\title{
Efectos de un sistema de aprendizaje tutorial online en profesorado tutor y de apoyo de las Islas Canarias para la instrucción temprana de las matemáticas en población de riesgo
}

\author{
Juan E. JIMÉNEZ, Nuria GUTIERREZ \\ Universidad de La Laguna, España
}

(Recibido 3 Marzo, 2016; Aceptado 18 Junio, 2016)

RESUMEN:El principal objetivo de este estudio ha sido analizar los efectos del programa tutorial Primate sobre los conocimientos y la valoración que realiza el profesorado tutor de aula y el profesorado de apoyo en las Islas Canarias. Se trata de un sistema de aprendizaje tutorial que utiliza la plataforma Moodle para crear un ambiente educativo virtual, dirigido para que el profesorado que atiende al alumnado en riesgo de presentar Dificultades Específica de Aprendizaje en Matemáticas (DEAM), pueda tener una formación sobre la instrucción temprana de las matemáticas en niños que se encuentran en situación de riesgo a partir de lo que prescribe la investigación científica. Se encontró que ambos grupos mejoraron sus conocimientos en las distintas áreas evaluadas a través del sistema tutorial. Finalmente, tanto el profesorado de apoyo como los profesores tutores de aula realizaron una valoración positiva de todos los módulos que componen el Primate.

Palabras clave: Enseñanza en línea; conocimientos del profesor, Modelo de Respuesta a la Intervención; formación del profesorado; instrucción directa; dificultades de aprendizaje en matemáticas.

Effects of Online Tutorial System on Classroom and Support In-Service Teachers in the Canary Islands for the Early Instruction of Students at Risk for Mathematics Difficulties

\begin{abstract}
The main objective of this study was to analyze the effects of the Primate online tutorial system on classroom and support in-service teachers in the Canary Islands for the early instruction of students at risk for mathematics difficulties. This is a tutorial learning system that uses the Moodle platform to create a virtual educational environment, aimed at teaching teachers that attends at-risk students who are considered to have a higher probability of failing academically in math. Teachers receive a training on how to instruct early mathematics in children who are at risk based on scientific research. A sample of classroom and support in-service teachers from the Canary Islands participated in the online course. It was found that both groups improved their knowledge in the different areas evaluated through the tutorial system. Finally, positive ratings were received by both the classroom and support in-service teachers.
\end{abstract}

Keywords: Web-based training; teacher knowledge, Response to Intervention Model, professional development; direct instruction; math learning disabilities. 
${ }^{1}$ Correspondencia:Facultad de Psicología, Campus de Guajara, 38200, Universidad de La Laguna, email: ejimenez@ull.es

En la actualidad, el énfasis en la atención a las Dificultades Específicas de Aprendizaje (DEA) debe centrarse en la detección e intervención temprana en los primeros cursos de Educación Primaria (LOMCE, 2013). Este enfoque viene apoyado por la evidencia científica que ha demostrado que los programas de prevención pueden reducir el número de alumnos identificados con DEA, y que, a largo plazo, resultan más eficaces que los programas de intervención que se implementan cuando la dificultad ya está instaurada (Lyon et al., 2001). En este contexto legislativo y educativo, desde la Comunidad Autónoma de Canarias se viene apostando por un modelo de Respuesta a la Intervención Temprana (Response to Intervention Model, RtI) (Jiménez, et al., 2010; 2011). Gracias, a su naturaleza multinivel, con el modelo RtI, se reduce el tiempo de espera del alumnado a la intervención (Fuchs y Fuchs, 2006). Los niveles 1, 2 y 3, difieren unos de otros en la estrategia instruccional, pasando de una intervención más general a una más específica, y en la ratio de alumnos, interviniendo con todo el grupo clase en el nivel 1, en pequeños grupos en el nivel 2 y de manera individual en el nivel 3. En este trabajo prestaremos especial atención a los niveles 2 y 3 de intervención, lo cual supone llevar a cabo una intervención de intensidad moderada (nivel 2) o intensa (nivel 3) basada en la evidencia científica (NCRTI, 2010), dirigida a estudiantes en riesgo de presentar Dificultades Específicas de Aprendizaje en Matemáticas (DEAM).

Este modelo, aunque no rechaza las diferencias individuales, pone énfasis en la importancia de la instrucción, ya que se deben adecuar los recursos pedagógicos a la respuesta del alumno a la instrucción (Speece y Case, 2001). Por lo tanto, para que el modelo pueda llevarse a la práctica de manera eficaz, la formación adecuada del profesorado es fundamental, dado que los beneficios propuestos dependen en gran medida de la adecuada implementación dentro de las escuelas (Brownell, Sindelar, Kiely y Danielson, 2010). La calidad en la formación del profesorado ha demostrado ser uno de los predictores más importantes del rendimiento académico de los alumnos (Dash et al., 2012; Haskins y Loeb, 2007). Sin embargo, en el caso de las matemáticas, tal y como señalan Alsina and Richard (2001), muchos profesores de Primaria y Secundaria consideran que su formación es insuficiente y no llegan a confiar plenamente en sus propios conocimientos.

La habilidad matemática es crítica para el aprendizaje, ya que, junto a la lectura y la escritura, es una de las habilidades instrumentales elementales (Orrantia, 2006). Numerosos estudios longitudinales han demostrado el impacto que tiene el aprendizaje temprano de las habilidades matemáticas sobre el rendimiento en cursos posteriores (v.gr., Aubrey, Godfrey, y Dahl, 2006; Jordan, Kaplan, Ramineni, y Locuniak, 2009). En España, la necesidad de la formación adecuada del profesorado en el área de matemáticas se ve reforzada por los informes internacionales que sitúan el rendimiento del alumnado español en el área de matemáticas muy por debajo de lo deseado en relación al resto de países que participaron en los estudios (i.e., MECD, 2012; OECD, 2014). El informe español del estudio TEDS-M (INEE, 2012) puso de manifiesto que la formación de los maestros de primaria en España sobre conocimientos matemáticos y didácticos era significativamente inferior al resto de países del estudio. Así, el estudio reveló que la formación del profesorado en el área de matemáticas es insuficiente.

En el caso específico del alumnado en riesgo de padecer DEAM, un aspecto 
fundamental es el empleo de prácticas de enseñanza basadas en la evidencia que hayan demostrado una mejora en el rendimiento matemático y la prevención de futuras dificultades (Bryant y Bryant, 2008). La intervención con el alumnado en riesgo de padecer DEAM o con el alumnado con bajo rendimiento en matemáticas, ha de ser explícita y sistemática. Uno de los componentes críticos en el desarrollo temprano de la competencia matemática es el concepto numérico (NationalMathematicsAdvisory Panel, 2008). Landerl, Bevan y Butterworth (2004) sugieren que el déficit central de las DEAM es un fallo a la hora de presentar y procesar los conceptos numéricos básicos, especialmente la idea de numerosidad, mostrando un rendimiento inferior a sus iguales en habilidades como el conteo, la comparación numérica y la subitización. Debido a este déficit en la comprensión del concepto numérico y sus propiedades, las habilidades de cálculo y resolución de problemas verbales pueden verse afectados (Blanco, 2007; Lembke y Foegen, 2009).

En relación con las tareas de cálculo, los alumnos con DEAM presentan dificultades en un uso efectivo de estrategias de cálculo y de conocimiento procedimental, la recuperación de los hechos numéricos, la conceptualización de la tarea y en la rapidez con la que realizan las operaciones (Geary, 2004). En el caso de la resolución de problemas verbales aritméticos, las dificultades para resolverlos están asociadas, además de al déficit en las habilidades computacionales, al uso de frases gramaticalmente o semánticamente complejas, a la necesidad de responder al problema con múltiples pasos, así como al empleo inadecuado de las estrategias de resolución de problemas (Rivera, 1997).

Los programas de formación online para el profesorado presentan múltiples ventajas con respecto a la formación presencial, ya se dispone de mayor autonomía y flexibilidad para decidir cuándo participar y beneficiarse del aprendizaje. Este tipo de formación permite superar los impedimentos que el horario laboral o el domicilio podrían suponer, permitiendo compatibilizar la situación laboral con la adquisición de nuevos conocimientos (Davis, 2009; LOMCE, 2013). Por lo tanto, a través de este tipo de formación el profesorado puede mantenerse actualizado y recibir una formación continua (Francis y Jacobsen, 2013).

Numerosos estudios han comparado los efectos de los programas online frente a los presenciales (i.e., Bernard et al., 2004; Jaggars y Xu, 2016). En el caso de las matemáticas, Russell, Kleiman, Carey y Douglas (2009) compararon los efectos de un programa de matemáticas realizado de forma presencial frente al mismo programa realizado vía online, sobre las creencias pedagógicas, las prácticas de instrucción y la comprensión de la enseñanza del sentido numérico y conceptos matemáticos relacionados, sin encontrar diferencias entre ambos, aunque los profesores que participaron en el curso online mostraron mayor interés por participar de nuevo en otros programas de formación. Ku et al (2011) encontraron que algunos de los factores que los profesores de matemáticas más valoraron de un programa de formación online fue la sensación de pertenencia a un grupo o comunidad, disponer de más asistencia para los problemas tecnológicos que pudieran surgir, y que la información acerca de los contenidos, objetivos, temporalización y evaluación se mostrasen de manera explícita.

Sin embargo, no toda la formación online puede ser considerada efectiva. Tal y como señalan Francis y Jacobsen (2004), a pesar de la gran cantidad de dinero destinada a la formación y desarrollo del profesorado, el aprendizaje suele presentarse fragmentado, superficial e inefectivo. Jiménez y Oshanahan (2016) señalan la importancia de diseñar programas de formación para el profesorado de calidad, basados en la evidencia científica, que 
sean capaces de proporcionar las herramientas adecuadas para desarrollar las habilidades del alumno. Por lo tanto, los programas de formación no han de centrarse exclusivamente en la trasmisión de información centrada en mejorar los conocimientos, sino que han de estar centrados en el desarrollo de habilidades (Jiménez et al., 2014). A pesar del creciente número de herramientas online dirigidas a la formación profesional del profesorado, en el caso de la enseñanza de las matemáticas, existen escasos estudios que hayan demostrado la efectividad de estos programas para los profesores y estudiantes. Ginsburg, Gray y Levin (2004) realizaron un metaanálisis donde revisaron un total de 40 programas online dirigidos a la formación profesional de los profesores de matemáticas. En su estudio concluyeron que a pesar de las ventajas que mostraban frente a la formación presencial, ningún estudio ofrecía datos acerca de su efectividad.

Para nuestro conocimiento, no existe ningún sistema tutorial dirigido al profesorado de Educación Infantil y primeros cursos de Educación Primaria, con el objetivo de facilitar una formación sobre como intervenir con el alumnado en riesgo de padecer DEAM en el contexto de implementación del modelo RtI. Este ha sido el objetivo a la hora de diseñar el sistema tutorial Primate, y ello ha sido posible gracias al apoyo del Plan Nacional I + D + i del Ministerio de Economía y Competitividad. En este trabajo se comparan los efectos que el programa Primate tiene sobre los conocimientos acerca del aprendizaje de las matemáticas temprana y sus dificultades entre el profesorado de apoyo y los profesores tutores. Además, se analiza el grado de satisfacción y aceptación con la formación online recibida a través del programa Primate.

\section{Método}

\section{Participantes}

Este estudio forma parte de un proyecto más amplio en el que se analizaron las prácticas de enseñanza de las matemáticas de una muestra representativa de profesores de las Islas Canarias. La población la formaban, por una parte, todos los maestros tutores $(\mathrm{N}=2373)$ de los cursos $1^{\circ}, 2^{\circ}$ y $3^{\circ}$ de Educación Primaria, y, por otra, todos los maestros de apoyo $(\mathrm{N}=1051)$ de todos los centros escolares de las Islas Canarias. El diseño del muestreo fue aleatorio simple. Se remitieron instrucciones precisas y detalladas a los tutores y de apoyo para la elección aleatoria. Con estas instrucciones se aseguró la estandarización del procedimiento y que las muestras eran realmente aleatorias y no dependían de ningún criterio ad hoc momentáneo. Los errores máximos en las estimaciones de los parámetros se calcularon con un error alfa del 5\%. Entonces para un universo de 2373 de profesores tutores, aplicando la corrección del tamaño muestral para poblaciones finitas, con un nivel de confianza del 95\% se obtuvo un tamaño muestral de aproximadamente 404. En el caso del profesorado de apoyo, para un universo de 1051, aplicando la corrección del tamaño muestral para poblaciones finitas, con un nivel de confianza del $95 \%$ se obtuvo un tamaño muestral de 316. De los 405 profesores tutores seleccionados al azar, el 56.0\% aceptó cumplimentar el cuestionario de prácticas de enseñanza $(\mathrm{N}=225)$, y de los 316 profesores de apoyo seleccionados al azar, el 51\% aceptó cumplimentar el cuestionario de prácticas de enseñanza $(\mathrm{N}=161)$. Una vez cumplimentado el cuestionario, se ofreció de forma voluntaria participar en el curso online y aceptó el $21.6 \%$ de profesorado tutor 
y el $20.2 \%$ del profesorado de apoyo. Por tanto, la muestra de estudio estaba formada por 88 profesores tutores y 64 profesores de apoyo de Educación Infantil y Primaria de las Islas Canarias. La cumplimentación del cuestionario general del programa Primate era opcional y fue contestada por 81 profesores que constituye el 53.3\% de la muestra, de los cuales un 39.4\% eran profesores tutores y un $13.8 \%$ eran profesores de apoyo.

\section{Procedimiento}

Una vez seleccionada la muestra de estudio, se llevaron a cabo sesiones previas a la formación con el objetivo de familiarizar a los usuarios en el uso de la plataforma online. Todos los participantes tenían acceso a un video de presentación y otro de demostración de cómo navegar por la plataforma, donde visualizaban la secuencia y pasos que a seguir. Se activaron dos tipos de foros en la plataforma, uno destinado a resolver dudas sobre los aspectos técnicos, donde los usuarios podían solicitar ayuda cuando tenían alguna dificultad para la navegación, y otro, centrado en los distintos tutoriales, donde los usuarios podían formular sus preguntas o dudas acerca de los contenidos que recibían en cada uno de los módulos del programa Primate. La navegación a través de los módulos no se realizaba de manera libre, sino que los usuarios avanzaban en la formación en función del calendario propuesto por semanas y las instrucciones de cada módulo. En los trabajos expuestos anteriormente, se ha comprobado que los profesores valoran positivamente este tipo de organización en programas de formación online (Bishop, Giles y Bryant, 2004; Ku et al., 2011). El primer módulo estaba formado por los distintos tutoriales de formación teórica. Antes de iniciar cada uno de los tutoriales, los participantes debían de rellenar el cuestionario de conocimientos (pretest), y una vez finalizado debían rellenar de nuevo el cuestionario de conocimientos del tutorial (postest) con el objetivo de evaluar los conocimientos adquiridos. Tras la finalización de los tutoriales del primer módulo, se activó el segundo módulo y así sucesivamente hasta completar el resto de módulos. Los usuarios tenían acceso libre a los tutoriales de formación teórica tras la finalización del primer módulo. Una vez transcurrido el periodo de cinco meses de formación se invitaba a los usuarios a completar el Cuestionario de Evaluación General del Programa Primate.

\section{Materiales}

\section{Programa Primate (primate.ull.es)}

Se trata de un sistema de aprendizaje tutorial que utiliza la plataforma Moodle para ofrecer al profesorado de Educación Infantil, de Educación Primaria y también el profesorado de apoyo, la formación y las herramientas necesarias para poder ofrecer una respuesta adecuada al alumnado que está en situación de riesgo de presentar DEAM, es decir, para poder intervenir con aquellos estudiantes que se encuentran en un nivel 2 y 3 del modelo RtI.

El programa está dividido en un total de 6 módulos. Un primer módulo (i.e., formación teórica) está dirigido a ofrecer los conocimientos teóricos esenciales basados en la evidencia científica que el profesorado que trabaja en los niveles iniciales de la enseñanza de las matemáticas debe conocer. Este primer módulo se divide en 7 tutoriales que aportan las herramientas necesarias para poder trabajar con el alumnado en riesgo de presentar DEAM. El primer tutorial (i.e., Dificultades específicas de aprendizaje en matemáticas y su prevención) se 
centra en la definición de las DEAM, criterios diagnósticos, la comprensión acerca de los déficits que subyacen a las DEAM, las características del alumnado en riesgo de presentar una DEAM, y ofrecer los conocimientos necesarios para poder prevenir de forma temprana. A través de un segundo tutorial (i.e., Principios básicos de la instrucción en las DEAM), se enseña a los profesores, cuáles son los componentes básicos que toda intervención debe reunir para tratar con el alumnado en riesgo de presentar DEAM. Esto es, la instrucción explícita, secuenciada e integrada, la instrucción basada en conceptos sólidos, la práctica y repetición, la diversidad de modalidades, y ejemplos de motivadores. Los 5 últimos tutoriales (i.e., Comprensión del número I y II, Cálculo I y II y Resolución de Problemas Verbales Aritméticos) están dirigidos a que el profesorado conozca cómo se adquieren y qué procesos cognitivos subyacen a las habilidades críticas que la evidencia científica prescribe para los niveles iniciales del aprendizaje de las matemáticas: dos tutoriales acerca de la comprensión del número (i.e., estrategias de conteo, estimación, codificación numérica, relaciones de orden como magnitud y distancia, relaciones de orden y transcodificación), dos tutoriales acerca del cálculo (i.e., definición de cálculo aritmético, suma y resta de un dígito, de más de un dígito, estimación en el cálculo, propiedades de la suma y la resta, y multiplicación) y un tutorial dirigido a la resolución de problemas verbales aritméticos (i.e., estructura semántica, lugar de la incógnita, etc.).

El segundo módulo (i.e., Implementación en las aulas) contiene material didáctico para el profesor y el alumno, con el objetivo de que el docente tenga los recursos didácticos necesarios para poder realizar la intervención de forma adecuada dentro del aula. Este módulo incluye 4 libros para el profesor y 4 cuadernos para el alumnado.

El tercer módulo (i.e., Evaluación) se centra en la formación del profesorado en el IPAM (Indicadores de Progreso de Aprendizaje en Matemáticas) (Jiménez y de León, 2016). Se trata de un instrumento de evaluación basado en el currículum (CBM, Curriculum Based Measure) que posee la doble finalidad de detectar el estatus de riesgo del alumno en tres momentos del curso escolar (inicio-medio-fin), y la evaluación del progreso de aprendizaje del alumno para poder adecuar las estrategias de intervención ante sus necesidades, tal y como prescribe el modelo RtI.

En el módulo 4 del programa (i.e., Experiencias), el profesor dispone una serie de grabaciones en vídeo sobre cómo llevar a cabo buenas prácticas de enseñanza cuando se quiere instruir de forma adecuada al alumnado que en los niveles iniciales ha sido detectado en situación de riesgo.

Por último, el módulo 5 (i.e., Recursos) ofrece bibliografía actualizada sobre la instrucción matemática fundamentada en la evidencia empírica, material didáctico, recursos multimedia, y legislación educativa.

\section{Cuestionario de Evaluación General del Programa Primate}

Este cuestionario consta de un conjunto de afirmaciones para conocer la valoración del usuario sobre el programa Primate. El cuestionario de valoración está integrado en el programa tutorial y comprende un total de nueve dimensiones con 10 ítems cada una que incluyen una escala de valoración tipo Likert 0 a 10: (1) fundamentación teórica (Tomo d) ₹( .92); (2) Estructura del programa de intervención (Tomo II) $(x=.96$ ); (3) Implementación del programa de intervención (Tomo IIIa): aspectos generales $(\alpha=.93)$; (4) Implementación del programa d e 
intervención (Tomo IIIb): aprendizaje del alumnado $(=.95)$; (5) Evaluađín del alumnado (Tomo IV) ( $\alpha=.96)$; (6) Experiencias (Tomo V) ( $\alpha=.96)$; (7) Recursos (Tomo VI) $(\alpha=.94$ ); (8) Innovación $(\alpha=.96)$; y (9) Beneficios y repercusión $(\alpha=.95)$.

Medidas sobre el conocimiento del profesorado

El programa Primate contiene un total de 7 cuestionarios pretest y postest, con 15 preguntas cada uno acerca de los distintos aspectos que se enseñan en cada uno de los tutoriales incluidos dentro del módulo de formación teórica: Dificultades específicas de aprendizaje en matemáticas y su prevención $(\alpha=.71)$; Principios básicos de la instrucción en las DEAM $(\alpha=$ .68); Comprensión del número (I) $(\alpha=.75)$; Comprensón del número (II) $(\alpha=.80)$; Cálculo (I) $(\alpha=.67)$; Cálculo (II) $(\alpha=.80)$; y Resolución de problemas verbales aritméticos $(\alpha=.70)$.

\section{Análisis de datos}

Para comparar los conocimientos adquiridos entre el profesorado de apoyo y el tutor del aula tras realizar la formación en el programa Primate se ha realizado un análisis factorial de medidas repetidas (ANOVA) a través del modelo lineal general (MGL), teniendo como factor intersujeto el grupo (profesor tutor de aula vs. profesor de apoyo), como factor intrasujeto el tiempo de medida (pre-post) y como variable dependiente las evaluaciones de los 7 módulos en los que se divide el programa (i.e., Tarea). Se comprobó el supuesto de esfericidad mediante la Prueba de esfericidad de Mauchly.

Con el fin de analizar la valoración general que realiza el profesorado de apoyo y el profesorado tutor del programa Primate, se generó una puntuación media global a partir de las medias en los distintos apartados del programa y se realizó un análisis de la varianza univariante (ANOVA). Para determinar si existían diferencias en la valoración realizada en los distintos apartados del programa se llevó a cabo un análisis multivariado (MANOVA) mediante el modelo lineal general (MLG), con un factor intersujeto: grupo (profesor tutor de aula vs. profesor de apoyo) y como variables dependientes las 9 dimensiones del cuestionario de evaluación del programa.

\section{Resultados}

\section{Conocimientos adquiridos}

La Tabla 1 muestra las puntuaciones medias pre- y postest de los 7 cuestionarios acerca de los conocimientos de las distintas áreas de formación teórica del Primate, antes y después de realizar los tutoriales. 
Tabla 1. Medias y desviaciones típicas de las puntuaciones pretest y posttest en los 7 tutoriales de formación teórica del programa Primate del profesorado de Apoyo Y profesorado Tutor de Aula

\begin{tabular}{|c|c|c|c|c|}
\hline \multicolumn{5}{|c|}{ Grupo } \\
\hline & \multicolumn{2}{|c|}{ Profesorado de apoyo } & \multicolumn{2}{|c|}{ Profesorado tutor } \\
\hline & Pretest & Postest & Pretest & Postest \\
\hline \multicolumn{5}{|c|}{ Dificultades específicas de aprendizaje en matemáticas y prevención } \\
\hline $\mathrm{M}$ & 3.47 & 7.14 & 3.00 & 6.37 \\
\hline DT & 1.22 & 1.74 & 1.10 & 1.81 \\
\hline \multicolumn{5}{|c|}{ Principios básicos de la instrucción en las DEAM } \\
\hline M & 5.69 & 7.81 & 5.38 & 7.26 \\
\hline DT & 1.49 & 1.19 & 1.36 & 1.70 \\
\hline \multicolumn{5}{|c|}{ Comprensión del número I } \\
\hline M & 4.92 & 7.16 & 4.56 & 7.36 \\
\hline DT & 1.40 & 1.63 & 1.15 & 1.39 \\
\hline \multicolumn{5}{|c|}{ Comprensión del número II } \\
\hline M & 4.90 & 8.18 & 4.55 & 8.18 \\
\hline DT & 1.88 & 1.37 & 1.37 & 1.07 \\
\hline \multicolumn{5}{|c|}{ Cálculo I } \\
\hline M & 5.88 & 7.02 & 5.61 & 6.84 \\
\hline DT & 1.52 & 1.21 & 1.34 & 0.96 \\
\hline \multicolumn{5}{|c|}{ Cálculo II } \\
\hline M & 6.92 & 9.11 & 6.85 & 8.51 \\
\hline DT & 0.10 & 0.94 & 1.42 & 1.59 \\
\hline \multicolumn{5}{|c|}{ Resolución de problemas verbales aritméticos } \\
\hline $\mathrm{M}$ & 5.52 & 7.50 & 5.19 & 7.56 \\
\hline DT & 1.29 & 1.19 & 1.33 & 1.44 \\
\hline
\end{tabular}

(Nota: M = Media; DT = Desviación Típica)

La prueba de esfericidad de Mauchly indica que la asunción de esfericidad fue violada para los efectos principales de Tarea, $\chi^{2}(20)=50.759, \mathrm{p}<.001$, y la inferaEarea $\mathrm{x}$ Momento $\chi^{2}(20)=40.676, \mathrm{p}<.01$. Por lo tanto, los grados de libertad fueron corregidos usando la estimación de esfericidad de Greenhouse-Geisser ( $€=.85$ para el efecto principal de Tarea y .88 para la interacción Tarea x Momento). Los resultados encontrados evidenciaron la existencia de un efecto principal en la variable Momento $F(1,106)=837.23, p<.001, \eta 2$ $=.88$. Se encontró además un efecto principal significativo de la variable Tarea, $\mathrm{F}(5.1,540.68)$ $=88.72, \mathrm{p}<.001, \eta 2=.86$ pero ambos estaban mediatizados por una interacción significativa Tarea $\mathrm{x}$ Momento F $(5.30,562.06)=30.03, \mathrm{p}<.001, \eta 2=.65$. Sin embargo, no existió interacción significativa entre las variables Momento $x$ Profesor $F(1,106)=.065, p=.80, \eta 2$ $=.001 \mathrm{ni}$ tampoco entre las variables Tarea $\mathrm{x}$ Profesor $\mathrm{F}(6,101)=1.10, \mathrm{p}=.364, \eta 2=.06$ (ver Figura 1) Además la interacción Tarea x Momento x Profesor, no alcanzó un valor significativo $\mathrm{F}(6,101)=1.48, \mathrm{p}=.19, \eta 2=.08$. 

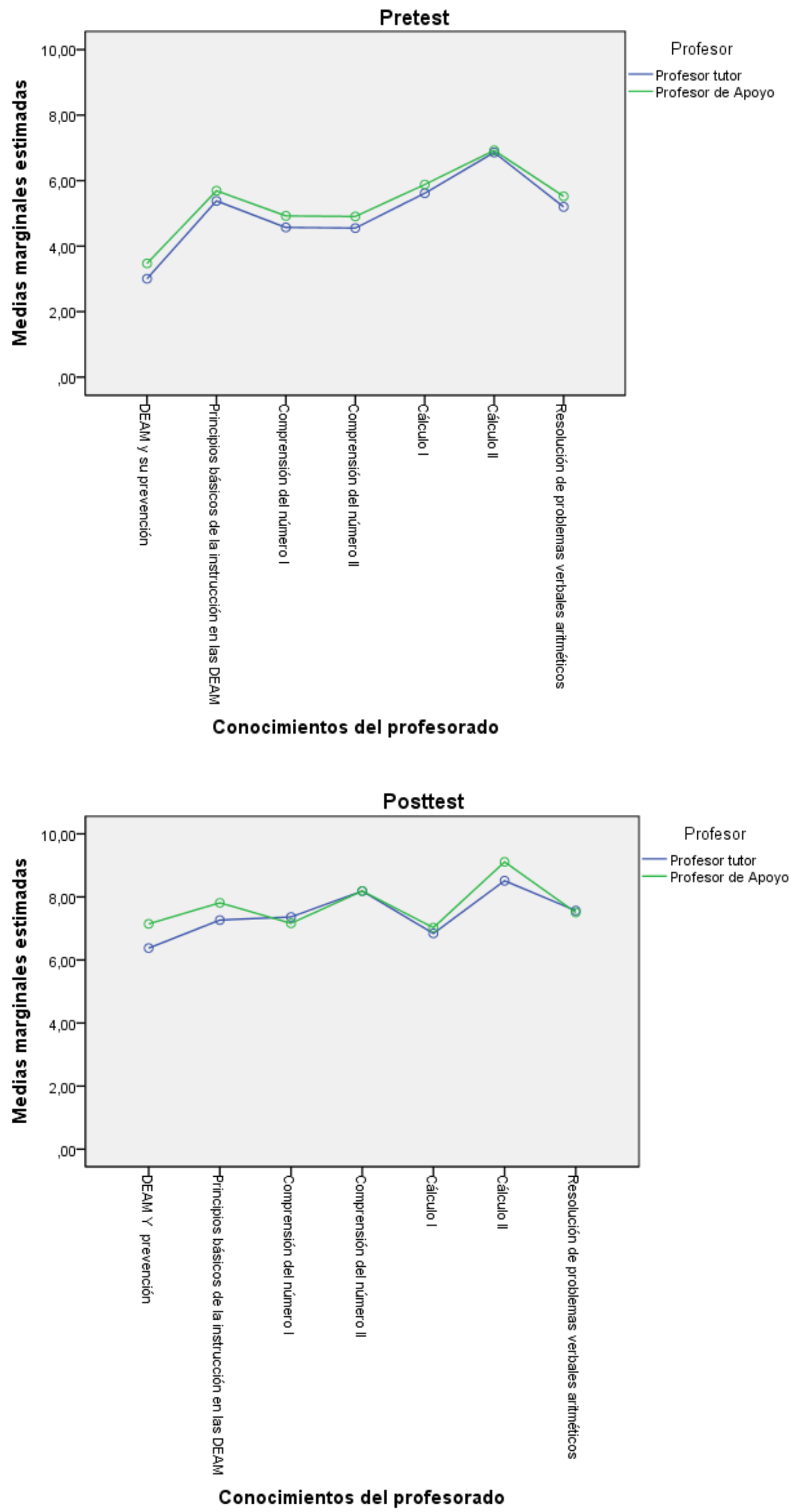

Figura 1. Medias de las puntuaciones pre y postest del profesor tutor de aula y de apoyo en las siete áreas del 
Primate.

\section{Valoración realizada}

La Tabla 2 muestra información referente a las medias y desviaciones típicas de las medidas de los distintos apartados del programa Primate evaluados por los profesores tutores y los profesores de apoyo.

Tabla 2.Medias y desviaciones típicas de las puntuaciones en la valoración de los distintos módulos del Programa Primate por parte del profesorado de Apoyo y Profesorado Tutor de

Aula

\begin{tabular}{|c|c|c|}
\hline \multicolumn{3}{|c|}{ Grupo } \\
\hline & Profesorado de apoyo & Profesorado tutor \\
\hline \multicolumn{3}{|c|}{ Fundamentación teórica (Tomo I) } \\
\hline $\mathrm{M}$ & 88.85 & 92.85 \\
\hline DT & 14.83 & 10.44 \\
\hline \multicolumn{3}{|c|}{ Estructura del programa de intervención (Tomo II) } \\
\hline M & 86.66 & 89.83 \\
\hline DT & 21.51 & 13.48 \\
\hline \multicolumn{3}{|c|}{$\begin{array}{l}\text { Implementación del programa de Intervención : } \\
\text { aspectos Generales (Tomo IIIa) }\end{array}$} \\
\hline $\mathrm{M}$ & 85.14 & 90.03 \\
\hline DT & 18.21 & 11.18 \\
\hline \multicolumn{3}{|c|}{$\begin{array}{l}\text { Implementación del programa de intervención: } \\
\text { aprendizaje del alumnado (Tomo IIIb) }\end{array}$} \\
\hline $\mathrm{M}$ & 86.90 & 91.81 \\
\hline DT & 19.34 & 11.36 \\
\hline \multicolumn{3}{|c|}{ Evaluación del alumnado (Tomo IV) } \\
\hline M & 85.71 & 91.81 \\
\hline & 17.67 & 12.97 \\
\hline \multicolumn{3}{|c|}{ Experiencias (Tomo V) } \\
\hline $\mathrm{M}$ & 85.61 & 92.58 \\
\hline DT & 20.87 & 13.49 \\
\hline \multicolumn{3}{|c|}{ Recursos (Tomo VI) } \\
\hline M & 84.76 & 88.91 \\
\hline DT & 19.74 & 13.02 \\
\hline \multicolumn{3}{|c|}{ Innovación } \\
\hline M & 81.90 & 88.30 \\
\hline DT & 18.79 & 15.43 \\
\hline \multicolumn{3}{|c|}{ Beneficios y repercusión } \\
\hline M & 80.52 & 86.53 \\
\hline DT & 16.77 & 14.21 \\
\hline
\end{tabular}

Los resultados del ANOVA no mostraron efectos significativos en la variable grupo $\mathrm{F}$ $(1,79)=2.360, \mathrm{p}=.128, \eta 2=.029$, en la valoración global del programa Primate. En relación a la valoración realizada para los distintos módulos del programa, el análisis MANOVA no reveló ninguna diferencia significativa entre el profesorado de apoyo y el profesorado tutor: 
fundamentación teórica (Tomo I) F $(1,79)=1,808, \mathrm{p}=.183, \eta 2=.022$; (2) Estructura del programa de intervención (Tomo II) F $(1,79)=.616$, p = .435, $\eta 2=.008$; (3) Implementación del programa de intervención (Tomo IIIa): aspectos generales $\mathrm{F}(1,79)=2.096, \mathrm{p}=.152, \eta 2=$ .026; (4) Implementación del programa de intervención (Tomo IIIb): aprendizaje del alumnado $\mathrm{F}(1,79)=1.964, \mathrm{p}=.165, \eta 2=.024 ;$ (5) Evaluación del alumnado (Tomo IV) $\mathrm{F}(1,79)=$ 2.828, $\mathrm{p}=.097, \eta 2=.035$; (6) Experiencias (Tomo V) F (1, 79) = 3.063, p = .084, $\eta 2$ = .037; (7) Recursos (Tomo VI) F (1, 79) =1.191, p = .278, $\eta 2=.015$; (8) Innovación F $(1,79)=$ 2.379, $\mathrm{p}=.127, \eta 2=.029 ; \mathrm{y}(9)$ Beneficios y repercusión $\mathrm{F}(1,79)=2.530, \mathrm{p}=.128, \eta 2=$ .116 (ver Figura 2).

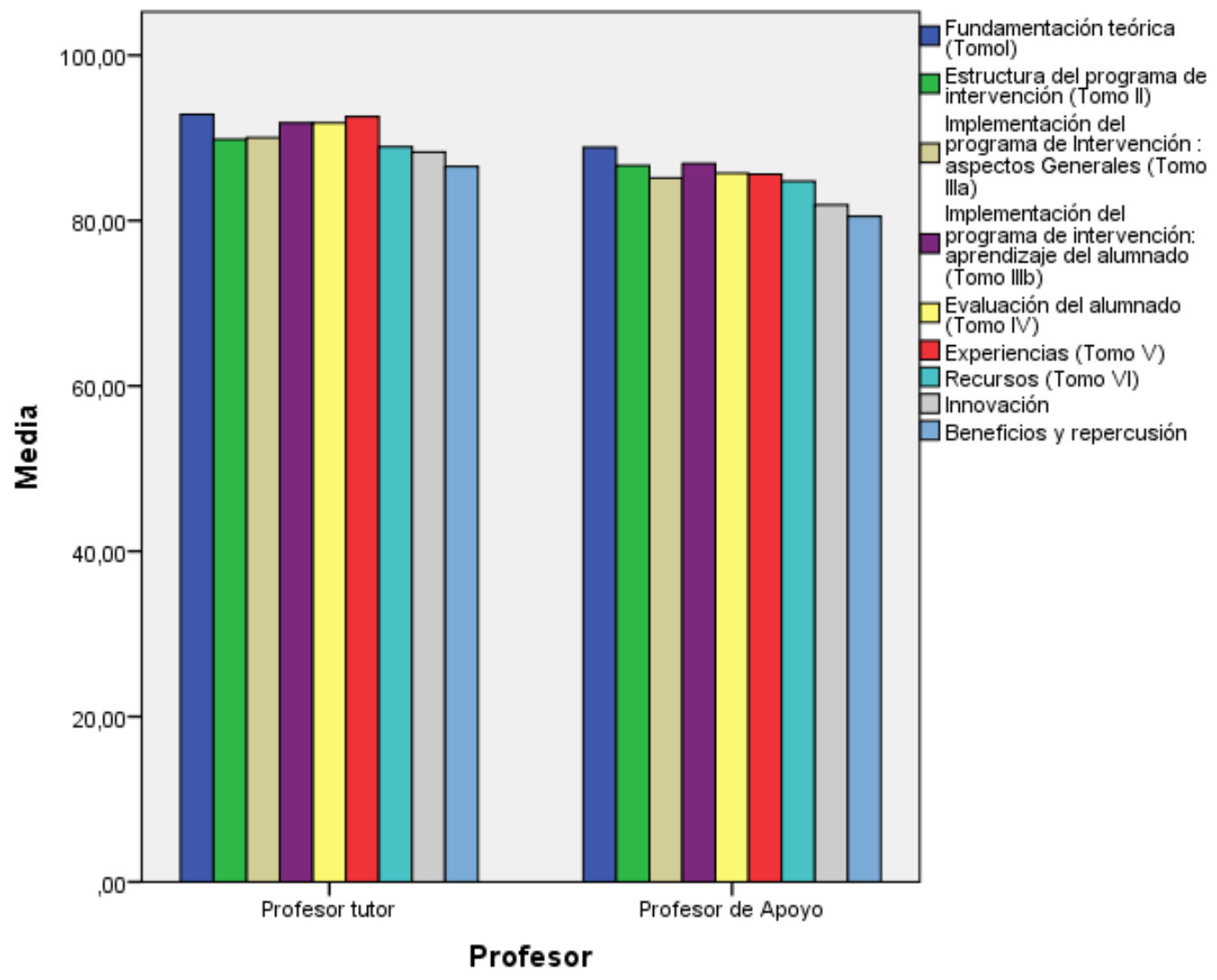

Figura 2. Medias de las puntuaciones en la valoración de los distintos módulos del programa Primate del profesorado de apoyo y profesorado tutor de aula.

\section{Discusión}

El primer objetivo de este estudio se centró en analizar y comparar los efectos sobre los conocimientos que profesores de apoyo y profesores tutores del aula adquieren a partir de la formación que ofrece el sistema tutorial Primate sobre las matemáticas temprana y su intervención con alumnado en riesgo de presentar DEAM. Los conocimientos especializados del profesorado sobre las matemáticas y su adecuada instrucción, han demostrado estar 
estrechamente relacionados con el rendimiento de los alumnos en los primeros años de Educación Primaria (Hill, Rowan y Ball, 2005). Sin embargo, los estudios anteriormente citados demuestran la falta de especialización del profesorado en el área de las matemáticas, y la escasez de programas de formación que se adecuen a las necesidades de aprendizaje, tanto de los alumnos como de los profesores.

Nuestro estudio reveló la falta de conocimientos, tanto del profesorado de apoyo como del profesorado tutor, en las distintas áreas implicadas en la enseñanza de las matemáticas temprana, en especial en aquellos conocimientos relacionados con el concepto de DEAM y su prevención. Tras la formación en el sistema tutorial Primate, los conocimientos en todas las áreas mejoraron de manera significativa, tanto en el profesorado tutor como en el profesorado de apoyo. Este hallazgo resulta positivo teniendo en cuenta que la atención al alumnado en riesgo de presentar DEAM (nivel 2 y 3 del modelo RtI) y otras NEAEs, debe realizarse en colaboración y coordinación entre ambos profesionales, tal y como establece el reglamento Orgánico de los centros docentes públicos no universitarios de la Comunidad Autónoma de Canarias (ORDEN de 9 de octubre de 2013).

Nuestros resultados coinciden con un estudio realizado por el equipo de Dash et al., (2012), donde analizaron el impacto sobre los conocimientos y las prácticas pedagógicas, de un curso online de 70 horas dirigido a la formación profesional de profesores de matemáticas. Sus resultados mostraron ganancias tanto en los conocimientos como en las prácticas de los profesores, aunque no encontraron diferencias en rendimiento de los estudiantes. Recientemente, Maloy, Edwars y Anderson (2010) demostraron la eficacia de un sistema tutorial de enseñanza de las matemáticas, el 4MALITY, dirigido a alumnos a partir de $3^{\circ}$ de Educación Primaria.

El segundo objetivo de este estudio fue comparar la satisfacción y aceptación de la formación en línea recibida a través del programa Primate. Tanto el profesorado de apoyo como el profesorado tutor, evaluaron todos los módulos del programa de manera positiva, sin existir diferencias entre profesores tutores y de apoyo en la valoración del programa. Por tanto, teniendo en cuenta los resultados obtenidos, podemos concluir que el programa Primate es efectivo a la hora de mejorar los conocimientos y ofrecer las herramientas necesarias para el profesorado de Educación Primaria y profesorado especialista que trabaja con el alumnado en riesgo de presentar DEAM, y por lo tanto puede ser empleado para la formación del profesorado en los niveles 2 y 3 del modelo RtI.

Uno de los principales objetivos que persigue la investigación científica es la transferencia de conocimiento a la sociedad y, en particular, al ámbito de la educación y la escuela. La escuela ha de ser un lugar donde las prácticas que allí acontecen deberían estar basadas en la evidencia científica. El trabajo que aquí presentamos es un ejemplo de ello ya que los hallazgos obtenidos se transfieren a la escuela en la Comunidad Autónoma de Canarias. El Plan Canario de Formación del Profesorado contempla entre sus líneas estratégicas "la atención a la diversidad" que tiene como propósito general ofrecer una formación especializada que facilite la detección, identificación e intervención temprana del alumnado que presenta necesidades específicas de apoyo educativo (NEAE). Con la finalidad de avanzar en el perfeccionamiento profesional, la Consejería de Educación y Universidades del Gobierno de Canarias define la acreditación en "El modelo RtI (Respuesta a la Intervención temprana): prevención y mejora del rendimiento en matemáticas”. En este proceso formativo se oferta la 
formación a través de la plataforma Primate y los recursos metodológicos que se proporciona en la formación para intervenir con el alumnado que es identificado en situación de riesgo.

\section{Agradecimientos}

Este trabajo ha sido posible gracias a la financiación del Plan Nacional I + D + i del Ministerio de Economía y Competitividad, con ref. EDU2012-35098, siendo el IP el primer autor, y a la beca concedida a la segunda autora financiada por la ACIISI y el Fondo Social Europeo.

\section{Referencias}

Alsina, C., y Richard, P. R. (2001). Reference levels in school mathematics education in Europe: National presentation Spain. European Mathematical Society. Recuperado de http://www.emis.de

Aubrey, C., Dahl, S., y Godfrey, R. (2006). Early mathematics development and later achievement: Further evidence. Mathematics Education Research Journal, 18(1), 27-46.

Blanco, M. (2009). Dificultades específicas del aprendizaje de las matemáticas en los primeros años de escolaridad: detección precoz y características evolutivas. Ministerio de Educación de España.

Brownell, M., Sindelar, P., Kiely, M., y Danielson, L. (2010). Special education teacher quality and preparation: Exposing foundations, constructing a new model. Exceptional Children, 76(3), 357-377.

Bryant, B. R., y Bryant, D. P. (2008). Introduction to special series: Mathematics and learning disabilities. Learning Disability Quarterly, 31(1), 3-10.

Dash, S., Magidin de Kramer, R., O’Dwyer, L. M., Masters, J., y Russell, M. (2012). Impact of Online Professional Development or Teacher Quality and Student Achievement in Fifth Grade Mathematics. Journal of Research on Technology in Education, 45(1), 1-26.

Davis, M. R. (2009). Creating value with online teacher learning. Education Week, 2 (2) 35-36.

Fuchs, L. S., y Fuchs, D. (2006). Identifying learning disabilities with RtI. Perspectives, 32, 39 $-43$.

Francis, K., y Jacobsen, M. (2013). Synchronous online collaborative professional development for elementary mathematics teachers. International Review of Research in Open and Distance Learning,14(3), 319-343.

Geary, C. (2004). Mathematics and learning disabilities. Journal of Learning Disabilities, 37, 4 -15 .

Ginsburg, A., Gray, T. y Levin, D. (2004) Online professional development for mathematics teachers: A strategic analysis. Washington D. C.: National Center for Technology Innovation, American Institutes for Research.

Haskins, R., y Loeb, S. (2007). A plan to improve the quality of teaching. Education Digest, 73 (1), 51-56.

Hill, H. C., Rowan, B., y Ball, D. L. (2005). Effects of teachers' mathematical knowledge for teaching on student achievement. American Education Research Journal, 42 (2), 371406. 
INEE (2012). TEDS-M. Estudio internacional sobre la formación inicial en matemáticas de los maestros. Informe español. Madrid: Ministerio de Educación, Cultura y Deporte.

Jaggars, S. S., y Xu, Di (2016). How do online course design features influence student performance? Computers and Education, 95, 270-284, doi: 10.1016/j.compedu.2016.01.014

Jiménez, J. E., Baker, D. L., Rodríguez, C., Crespo, P., Artiles, C. y Alfonso, M. (2011). Evaluación del progreso de aprendizaje en lectura dentro de un Modelo de Respuesta a la Intervención (RtI) en la Comunidad Autónoma de Canarias. Escritos de Psicología, 4, 56-64.

Jiménez, J.E., y de León, S. (2016). Indicadores de progreso de aprendizaje en matemáticas (IPAM). Universidad de La Laguna.

Jiménez, J. E. y O'Shanahan, I. (2016). Effects of web-based training on Spanish pre-service and in-service teacher knowledge and implicit beliefs on learning to read. Teaching and Teacher Education, 55, 175-187. doi: http://dx.doi.org/10.1016/j.tate.2016.01.006

Jiménez, J. E., O’Shanahan, I., González, J. A., Frugone, M., y Barrientos, P. (2014). Preservice teachers and in-service teachers' rating of the Letra program: a piloting experience in Latin American countries. Estudios de Psicología, 35, 605-624. doi: 10.1080/02109395.2014.974423

Jiménez, J. E., Rodríguez, C., Crespo, P., González, D., Artiles, C., y Alfonso, A. (2010). Implementation of Response to Intervention (RtI) Model in Spain: An example of a collaboration between Canarian universities and the department of education of the Canary Islands. Psicothema, 22 (4), 935-942.

Jordan, N. C., Kaplan, D., Locuniak, M., y Ramineni, C. (2007). Predicting first-grade math achievement from developmental number sense. Learning Disabilities Research y Practice, 22 (1), 36-46.

Jordan, N. C., Kaplan, D., Ramineni, C., y Locuniak, M. N. (2009). Early math matters: Kindergarten number competence and later mathematics outcomes. Developmental Psychology, 45 (3), 850-867.

Ku, H., Akarasriworn, C., Glassmeyer, D. M., Mendoza, B., y Rice, L. A. (2011). Teaching an online graduate mathematics education course for in-service mathematics teachers. Quarterly Review of Distance Education, 12 (2), 135-147.

Landerl, K., Bevan, A., y Butterworth, B. (2004). Developmental dyscalculia and basic numerical capacities: A study of 8-9-year-old students. Cognition, 93, 99-125.

Lembke, E., y Foegen, A. (2009). Identifying early numeracy indicators for kindergarten and first-grade students. LearningDisabilitiesResearch y Practice, 24, 12-20.

LOMCE, Ley Orgánica 8/2013, de 9 de diciembre, para la mejora de la calidad educativa. Boletín Oficial del Estado, $n^{\circ}$ 295, 2013, 10 diciembre.

Lyon, G. R., Fletcher, J. M. Shaywitz, S. E., Shaywitz, B. A., Wood, F. B., Schulte, A., Olson, R. K., y Torgesen, J. K. (2001). Learning disabilities: An evidence-based conceptualization. Rethinking special education for a new century (pp. 259-287). Washington, D.C.: Fordham Foundation and Progressive Policy Institute.

Maloy, R. W., Edwards, S. A. y Anderson, G. (2010). Teaching math problem solving using a web-based tutoring system, learning games, and students' writing. Journal of STEM Education, 11, 82-89. 
Ministerio de Educación, Cultura y Deporte (2012). PIRLS - TIMSS 2011. Estudio Internacional de progreso en comprensión lectora, matemáticas y ciencias. Informe español. Volumen I. Madrid: Instituto Nacional de Evaluación Educativa.

National Center on Response to Intervention (2010). Information Brief: Developing an RTI Guidance Document.

National Mathematics Advisory Panel (2008). Foundations for Success:The Final Report of the National Mathematics Advisory Panel.U.S. Department of Education: Washington, DC.

OECD (2014). PISA 2012 Results: What students know and can do - student Performance in Mathematics, Reading and Science (Volume I, Revised edition, February 2014), PISA, OECD.

ORDEN de 9 de octubre de 2013, por la que se desarrolla el Decreto 81/2010, de 8 de julio, por el que se aprueba el Reglamento Orgánico de los centros docentes públicos no universitarios de la Comunidad Autónoma de Canarias, en lo referente a su organización y funcionamiento.

Orrantia J. (2006). Dificultades en el aprendizaje de las matemáticas: una perspectiva evolutiva. Psicopedagogia, 71, 150-80.

Rivera, D. P. (1997). Mathematics education and students with learning disabilities: Introduction to special series. Journal of Learning Disabilities, 30 (2), 2-19.

Russell, M., Kleiman, G., Carey, R. y Douglas, J. (2009). Comparing self-paced and cohortbased online courses for teachers. Journal of Research on Technology in Education, 41(4), 443-466.

Speece, D. L., y Case, L. P. (2001). Classification in context: An alternative approach to identifying early reading disability. Journal of Educational Psychology, 93, 735-749. 